\title{
COMPLICAÇÕES DECORRENTES DO USO DE ÂNCORAS METÁLICAS EM ARTROSCOPIAS DE OMBRO
}

\author{
COMPLICATIONS RESULTING FROM THE USE OF METAL \\ ANCHORS IN SHOULDER ARTHROSCOPY
}

Glaydson Gomes Godinho', Flavio Oliveira França², José Marcio Alves Freitas ${ }^{3}$, Paulo Nascimento Aguiar4, Marcelo de Carvalho Leite ${ }^{4}$

\section{RESUMO}

Objetivo: Identificar as complicações referentes ao uso de âncoras metálicas nos procedimentos artroscópicos do ombro. Métodos: No período de dezembro de 1997 a agosto de 2007, 28 ombros de 28 pacientes (23 do sexo masculino e cinco do feminino) foram reoperados nos Hospitais Ortopédico, Belo Horizonte e da Polícia Militar, em Belo Horizonte, MG, devido a complicações como soltura de âncoras e âncoras proeminentes. As cirurgias primárias tiveram como objetivo tratar 20 instabilidades anteriores traumáticas (71,5\%), uma instabilidade posterior (3,5\%), uma lesão slap (3,5\%) e seis lesões do manguito rotador (21,5\%). Foram utilizadas a classificação radiográfica de Samilson e Prieto e a artroscópica de Outerbridge na avaliação do grau de artrose dos pacientes. Na avaliação dos pacientes foram usados os critérios do índice da UCLA (University of Califórnia at Los Angeles). Resultados: Em todos os pacientes tratava-se de revisões artroscópicas. Em dois casos, após a retirada das âncoras havia sinais clínicos de instabilidade, optando-se, então, pela estabilização aberta pela técnica de Latarjet-Patte. Conclusões: As complicações com âncoras de sutura metálicas são decorrentes do emprego inadequado da técnica cirúrgica em artroscopia.

Descritores - Articulação do ombro; Artroscopia; Âncoras ósseas

\section{ABSTRACT}

Objective: to identify the complications concerning the use of metal anchors in shoulder arthroscopic procedures. Methods: 28 shoulders of 28 patients (23 male and 5 female) have been re-operated in the period between December 1997 and August 2007, at Hospital Ortopédico, Belo Horizonte Hospital and Military Police Hospital in Belo Horizonte, MG, as a result of complications such as loose anchors and prominent anchors. The primary surgeries intended to treat 20 anterior traumatic instabilities (71.5\%), one posterior instability (3.5\%), one slap injury (3.5\%), six procedures for treating injuries on the rotator cuff (21.5\%). We used the X-ray classification suggested by Samilson and Prieto and Outerbridge arthroscopic classification for assessing patients' degree of arthrosis. All patients were evaluated by the UCLA (University of California at Los Angeles) index criteria. Results: In all patients, arthroscopic reviews were made. In two cases, after anchors removal, clinical signs of instability were seen, leading to the decision of providing open stabilization by Latarjet-Patte technique. Conclusion: the complications with metallic-suture anchors result from inappropriate surgical techniques applied in arthroscopy.

Keywords - Shoulder joint; Arthroscopy; Bone anchors

1 - Chefe do Grupo do Ombro do Hospital Ortopédico e Hospital Belo Horizonte,

2 - Cirurgião Assistente do Grupo de Ombro do Hospital Ortopédico e Hospital da Polícia Militar de Minas Gerais.

3 - Cirurgião Assistente do Grupo de Ombro do Hospital Ortopédico e Hospital Belo Horizonte.

4 - Estagiários do Grupo de Ombro do Hospital Ortopédico e Hospital Belo Horizonte.

Trabalho realizado no Hospital Ortopédico pelo Núcleo do Grupo do Ombro.

Correspondência: Rua Prof. Otávio Coelho de Magalhães, 111 - Mangabeiras - 30210-300 - Belo Horizonte, MG. E-mail: ggodinho@terra.com.br 


\section{INTRODUÇÃO}

O lábio ântero-inferior está inserido na margem da cavidade glenoide, aumentando sua profundidade e auxiliando na estabilização da cabeça do úmero ${ }^{(1)}$. Em 1997, Koss et al $^{(2)}$ descreveram a reparação da lesão de Bankart usando sutura metálica. Foram avaliados 26 pacientes sem complicações relacionadas com o dispositivo. Com a evolução das técnicas artroscópicas para tratamento das lesões no ombro, as âncoras de sutura foram sendo aperfeiçoadas. Existe consenso na literatura de que a resistência das âncoras de sutura é relacionada com o tipo de osso (osteoporose, porosidade cortical) ${ }^{(3)}$. Zuckerman e Matsen ${ }^{(4)}$ classificaram as complicações, de acordo com a causa, em: $1^{\circ}$ ) colocação incorreta do implante; $2^{\circ}$ ) migração; $3^{\circ}$ ) soltura (perda); $4^{\circ}$ ) quebra. Tais complicações têm potencial para causar erosão condral e osteoartrose, consequências extremamente danosas à articulação glenoumeral.

O objetivo deste trabalho é identificar as complicações mais comuns, decorrentes do uso de âncoras metálicas em artroscopias do ombro. As consequências tardias da osteoartrose não são objeto do estudo.

\section{MÉTODOS}

Foram submetidos à revisão artroscópica 28 pacientes (28 ombros) acometidos por complicações decorrentes do uso de âncoras metálicas. Todos os casos foram reoperados: no Hospital Ortopédico, no Hospital Belo Horizonte e no Hospital da Polícia Militar em Belo Horizonte, MG, pelos três cirurgiões do grupo.

A idade variou entre 19 e 69 anos, com média de 35,2 anos. Vinte e três pacientes eram do sexo masculino e cinco, do feminino.

O lado direito foi acometido 18 (64,3\%) vezes e o esquerdo, 10 (35,7\%). O lado dominante foi reoperado 17 (60,7\%) vezes. Após cirurgia primária, os sintomas mais frequentemente observados foram: dor, limitação de amplitude de movimentos (ADM) e, principalmente nos procedimentos intra-articulares, crepitação à manobra de Jobe (abdução acima de $90^{\circ}$ contra resistência, no plano frontal, com membros superiores rodados internamente: teste do supra-espinal) ${ }^{(5)}$. Esses sintomas foram detectados num período médio de 19,2 meses, variando de dois a 52 meses. Em 22 pacientes (78,5\%) esse sintomas foram identificados em média de 5,3 meses. Vinte e sete meses foi o período necessário para a detecção dos sintomas clínicos em seis pacientes (21,5\%). Ao avaliarmos o intervalo de tempo entre a primeira cirur- gia e a revisão, encontramos um período médio de 20,5 meses, variando de quatro a 52 meses. Em 22 pacientes (78,5\%) esse período médio foi de 7,09 meses e, em seis pacientes, de 28 meses.

O período de seguimento médio foi de 37 meses, variando de sete a 108 meses.

Nosso estudo apresenta 20 instabilidades anteriores, uma instabilidade posterior, uma lesão slap e seis revisões de reparos de manguito rotador, sendo $75 \%$ dos casos intra-articulares e 25\% extra-articulares.

Todos pacientes foram avaliados conforme os critérios do índice da UCLA (University of California at Los Angeles) ${ }^{(6)}$. Foi usada a classificação de Outerbridge ${ }^{(7)}$ (Tabela 1) na avaliação artroscópica, para determinar o grau de lesão condral e, a classificação radiográfica de Samilson e Prieto ${ }^{(8)}$, para artrose glenoumeral.

Tabela 1 - Classificação de Outerbridge

\begin{tabular}{l|l}
\hline Grau I & Amolecimento da cartilagem \\
\hline Grau II & Fragmentação e fissura de uma área de $1,5 \mathrm{~cm}$ ou menos de diâmetro. \\
\hline Grau III & Fragmentação e fissura de uma área maior que 1,5cm de diâmetro. \\
\hline Grau IV & Erosão da cartilagem subcondral. \\
\hline
\end{tabular}

As revisões foram realizadas na posição de decúbito lateral, com portais posterior, ântero-superior e ânteroinferior para procedimentos intra-articulares e portais posterior, ântero-superior e lateral nos extra-articulares. Todos os procedimentos constaram de retirada ou sepultamento de âncoras inadequadas, sinovectomia e bursectomia. Em dois pacientes, depois da retirada de âncoras por via artroscópica, foi constatado que ainda havia sinais de instabilidade. Optamos, nesses casos de instabilidade residual, pela estabilização glenoumeral aberta, pela técnica de Patte e Debeyre ${ }^{(9)}$.

Os procedimentos, diagnóstico inicial e cirurgias realizadas estão resumidos na tabela 2 .

Todos os casos intra-articulares foram submetidos à liberação de aderências e sinovectomia e, em todos os extra-articulares, foi feita bursectomia.

\section{RESULTADOS}

Nas 28 cirurgias primárias foram utilizadas 82 âncoras metálicas, com média de 2,9 âncoras por paciente.

Quarenta e sete âncoras (57,31\%) estavam inadequadas em seus posicionamentos (41 intra-articulares e seis extra-articulares). Do total de âncoras inadequadas, 43 foram retiradas e quatro, sepultadas. 
Tabela 2 - Sequência de pacientes segundo o diagnóstico, cirurgia primária e revisão artroscópica, enfatizando a posição da âncora inadequada na glenoide em comparação com os números de mostradores de um relógio. (1, 3, 5h para ombro direito e 7, 9, 11h para o esquerdo)

\begin{tabular}{|c|c|c|c|c|c|}
\hline $\begin{array}{l}N^{0} \text { sequencial } \\
\text { dos pacientes }\end{array}$ & Diagnóstico & Cir. inicial & Âncora retirada & Âncora sepultada & Outros procedimentos \\
\hline 1 & Lesão MR & Reparo com 2 Rotax & 1 âncora Rotax & & Reparo MR + tenotomia CLB \\
\hline 2 & Lesão MR & Reparo com 2 Rotax & 1 âncora Rotax & & Reparo MR \\
\hline 3 & Lesão MR & Reparo com 3 Rotax & 1 âncora Rotax & & Reparo MR \\
\hline 4 & Lesão MR & Reparo com 2 Revo & 1 âncora Revo & & \\
\hline 5 & Lesão MR & Reparo com 2 Revo & 1 âncora Revo & & \\
\hline 6 & Lesão MR & Reparo com 2 Revo & 1 âncora Revo & & \\
\hline 7 & SLAP & Reparo 2 mini- Rotax & 2 mini- Rotax (11 e 1h) & & Tenotomia CLB \\
\hline 8 & IAT & Reparo 3 mini- Rotax & 2 mini- Rotax (5 e 3h) & & Microfraturas \\
\hline 9 & IAT & Reparo 3 mini- Rotax & 1 mini- Rotax (5h) & 2 mini-Rotax (3 e 1h) & \\
\hline 10 & IAT & Reparo 3 Rotax & 3 Rotax (5, 3 e $1 \mathrm{~h})$ & & \\
\hline 11 & IAT & Reparo 3 Rotax & 3 Rotax (5, 3 e $1 h)$ & & Novo reparo da lesão de Bankart \\
\hline 12 & IAT & Reparo 3 Revo & 1 Revo (7h) & & \\
\hline 13 & IAT & Reparo 4 mini- Rotax & 2 mini- Rotax (11 e 9h) & & Latarjet-Patte \\
\hline 14 & IAT & Reparo 3 Revo & 3 Revo (11, 9 e $7 \mathrm{~h})$ & & Ressecção osteófito cab. umeral \\
\hline 15 & IAT & Reparo 2 mini- Rotax & 2 mini- Rotax (5 e 3h) & & Ressecção de osteófitos \\
\hline 16 & IAT & Reparo 4 mini- Rotax & 1 mini- Rotax (3h) & 1 mini-Rotax (5h) & \\
\hline 17 & IAT & Reparo 3 mini- Rotax & 1 mini- Rotax ( 7h) & & Radiofrequência + microfraturas \\
\hline 18 & IAT & Reparo 4 mini- Rotax & 1 mini- Rotax (5h) & & \\
\hline 19 & IAT & Reparo 4 mini- Revo & 1 mini- Revo (3h) & & \\
\hline 20 & IAT & Reparo 4 mini- Revo & 1 mini- Revo (7h) & & \\
\hline 21 & IAT & Reparo 3 mini- Revo & 2 mini- Revo (5 e $3 h$ ) & 1 mini-Revo (1h) & \\
\hline 22 & IAT & Reparo 3 mini- Rotax & 2 mini- Rotax (3 e 1h) & & \\
\hline 23 & IAT & Reparo 3 mini- Rotax & 1 mini- Rotax (5h) & & Latarjet-Patte \\
\hline 24 & IAT & Reparo 3 mini- Revo & 3 mini- Revo (5, 3 e $1 \mathrm{~h})$ & & \\
\hline 25 & IA & Reparo ???? & 1 âncora (5h) & & \\
\hline 26 & IAT & Reparo 3 mini- Revo & 1 mini- Revo (5h) & & \\
\hline 27 & IAT & Reparo 3 mini- Revo & 1 mini- Revo (7h) & & \\
\hline 28 & IPT & Reparo 3 Rotax & 2 Rotax (3 e 5h) & & \\
\hline
\end{tabular}

$\mathrm{MR}$ = manguito rotador; $\mathrm{IAT}$ = instabilidade anterior traumática; $\mathrm{CLB}$ = cabeça longa do bíceps; IPT = instabilidade posterior traumática

Foram retiradas 100\% das âncoras extra-articulares inadequadas e 90,2\% (37) das âncoras intra-articulares. O maior número de âncoras intra-articulares que complicaram estava nas posições inferiores: 5 h (31,70\%) e 3h (26,82\%) (Tabela 3). Apenas quatro (9,75\%) âncoras foram sepultadas (Tabela 4).

Tabela 3 - Número de âncoras intra-articulares complicadas em relação com a posição

\begin{tabular}{c|c|c|c|c|c}
\hline $1 \mathrm{~h}$ & $3 \mathrm{~h}$ & $5 \mathrm{~h}$ & $7 \mathrm{~h}$ & $9 \mathrm{~h}$ & $11 \mathrm{~h}$ \\
\hline $7(17,0 \%)$ & $11(26,8 \%)$ & $13(31,7 \%)$ & $5(12,2 \%)$ & $2(4,9 \%)$ & $11(7,3 \%)$ \\
\hline
\end{tabular}

Tabela 4 - Âncoras retiradas e sepultadas em relação com a posição

\begin{tabular}{l|c|c|c|c|c|c}
\hline & 1h & $3 \mathrm{~h}$ & $5 \mathrm{~h}$ & $\mathbf{7 h}$ & $9 \mathrm{~h}$ & $\mathbf{1 1 h}$ \\
\hline Retiradas & 5 & 10 & 12 & 5 & 2 & 3 \\
\hline Sepultadas & 2 & 1 & 1 & & & \\
\hline
\end{tabular}

Nove pacientes apresentavam artrose glenoumeral segundo a classificação de Samilson e Prieto ${ }^{(8)}$ (Tabela 5).

\section{Tabela 5}

\begin{tabular}{c|c|c|c|c}
\hline No pacientes & Tipo I & Tipo II & Tipo III & Tipo IV \\
\hline 9 & 1 & 3 & 3 & 2 \\
\hline
\end{tabular}

Todos os pacientes apresentavam algum grau de lesão condral. Dezenove pacientes (67,8\%) mostravam graus mais graves de acometimento (III e IV), segundo Outerbridge $^{(5)}$ (Tabela 6).

\section{Tabela 6}

\begin{tabular}{c|c|c|c|c}
\hline No pacientes & Tipo I & Tipo II & Tipo III & Tipo IV \\
\hline 28 & \multicolumn{2}{|c|}{8} & 9 & 10 \\
\hline
\end{tabular}


Após revisão artroscópica, os pacientes foram avaliados conforme os critérios de pontuação do índice da UCLA ( Tabela 7).

Tabela 7

\begin{tabular}{l|c|c}
\hline Índice da UCLA & Pacientes & $\%$ \\
\hline $\mathbf{3 4}$ - 35 (excelente) & 6 & 21,4 \\
\hline $\mathbf{2 8}-\mathbf{3 3}$ (bom) & 15 & 53,6 \\
\hline $\mathbf{2 1} \boldsymbol{- 2 7}$ (regular) & 5 & 17,8 \\
\hline $\mathbf{0}-\mathbf{2 0}$ (ruim) & 2 & 7,1 \\
\hline
\end{tabular}

\section{DISCUSSÃO}

Complicações com uso de materiais metálicos no ombro são descritas em alguns trabalhos da literatura; porém, as descrições de complicações com o uso de âncoras metálicas em artroscopias de ombro são muito escassas.

Zuckerman e Matsen ${ }^{(4)}$ estudaram 37 pacientes com complicações na articulação glenoumeral relacionadas com o uso de parafusos ou grampos após cirurgia aberta. Dez dos 37 pacientes tiveram alterações erosivas da cavidade glenoide ou da cabeça umeral diretamente relacionadas com a colocação incorreta do dispositivo de fixação. Quatorze pacientes tiveram perda permanente da função glenoumeral.

Kaar et $\mathrm{al}^{(3)}$ observaram oito pacientes com complicações após cirurgia aberta, na qual foram utilizadas âncoras metálicas de sutura. Eles relataram que três pacientes (38\%) desenvolveram dano articular grave diretamente causado por uma âncora metálica exposta ou solta.

Ejnisman et al $^{(10)}$ estudaram oito pacientes com complicações devidas ao uso de âncoras em cirurgias abertas (três pacientes) e artroscópicas (cinco pacientes). Nesse estudo, $100 \%$ dos casos apresentavam lesão condral da cabeça umeral e $80 \%$ da cavidade glenoide.

Nosso estudo mostra complicações com o uso de âncoras metálicas em procedimentos exclusivamente artroscópicos (100\% dos casos). Do total de 28 pacientes, 22 foram submetidos a procedimentos articulares e apresentavam danos condrais glenoumerais em graus variados da classificação de Outerbridge ${ }^{(7)}$, prevalecendo em 19 desses casos (86,3\%) os graus III e IV. Os restantes seis casos referem-se a procedimentos extra-articulares. Âncoras malposicionadas e expostas foram a causa desses danos.

Koss et $\mathrm{al}^{(2)}$ evidenciaram um caso em que, algumas semanas após estabilização aberta de uma lesão de Bankart, havia presença de dor e crepitação crescente. Os sintomas eram mais observados em abdução e rotação interna do braço.
Em nossa série constatamos que os sintomas mais comuns eram dor e crepitação, que se agravavam com o braço em abdução de 90 graus e rotação interna, especialmente quando os pacientes eram solicitados a resistir à força do examinador em sentido contrário, para baixo (posição do teste de Jobe para avaliação da força do músculo supra-espinal). A limitação da amplitude de movimentos (ADM) foi um sinal frequente.

Rhee et $\mathrm{al}^{(1)}$ relataram a realização da segunda cirurgia em média de 12 meses, após a cirurgia primária.

Ejnisman et $\mathrm{al}^{(10)}$ relataram que, dos oito pacientes apresentados, apenas um (12,5\%) foi revisado nas primeiras seis semanas; o restante, após três meses de evolução.

Os dados da literatura, em relação ao tempo de revisão são divergentes. Neste estudo, o período médio observado entre a primeira e a segunda cirurgias foi de 20,5 meses. Constatamos que, do total de 28 pacientes, 22 (78,5\%) tiveram média de 7,1 meses e que, em seis pacientes (21,5\%), essa média subiu para 28 meses ou mais. Esse dado coincide com a observação de que esses seis pacientes, com média mais alta, estavam entre os sete casos com resultados regulares e ruins pelo índice da UCLA.

Realizamos uma avaliação funcional em curto prazo levando-se em conta os critérios do índice da UCLA. Observamos 75\% de excelentes e bons resultados. Vale salientar que nosso estudo aborda uma complicação catastrófica e irreversível e que os dados obtidos nessa avaliação precoce traduzem melhora principalmente nos critérios de dor e satisfação do paciente, o que elevou a pontuação final. Acreditamos que esses números provavelmente serão menos favoráveis com o tempo, em função da artrose que tende a evoluir.

O paciente $n^{\circ} 9$ apresentou índice da UCLA 14 (ruim) em função de neurapraxia axilar associada como complicação pós-cirúrgica do primeiro procedimento.

Nove pacientes do estudo apresentavam algum grau de artrose (Tabela 5), segundo a classificação radiográfica de Samilson e Prieto ${ }^{(8)}$. Como essa classificação é radiográfica e para que haja alterações é necessária cronicidade da doença, observamos que as alterações encontradas em nossos pacientes correspondem àqueles seis que tiveram um período médio entre a primeira e segunda cirurgia igual ou superior a 28 meses e àqueles que já apresentavam sinais radiográficos de artrose antes da primeira cirurgia. 
Uma constatação importante de nosso estudo é que das 41 âncoras intra-articulares inadequadas, 24 (38,5\%) se encontravam nas posições de 3 e 5 horas (Tabela 3 ). Esses dados sugerem que a causa possa ser provável dificuldade técnica ou material de qualidade questionável, que poderia ter tornado mais difícil o acesso à porção mais distal da cavidade glenoide.

Rhee et al ${ }^{(1)}$ alertaram para os cuidados intraoperatórios em termos de colocação de âncoras para reduzir a probabilidade de solturas: $1^{\circ}$ ) A primeira âncora é fundamental para estabelecer a tensão capsular apropriada e deve ser colocada na posição de 5 h (para o ombro direito) ou 7h (para o ombro esquerdo) em relação à cavidade glenoide; $2^{\circ}$ ) O orifício de introdução da âncora deve estar situado na face articular da cavidade glenoide, a 1 a 2mm de sua margem, com inclinação média de 45 graus até que a marca indicadora da introdução tenha ultrapassado o osso cortical; pode ser usada força suave póstero-inferior sobre a cabeça do úmero com o próprio introdutor, como "uma técnica de movimento de braço de alavanca”, o que facilita a colocação da âncora com a melhor inclinação; $3^{\circ}$ ) A âncora deve ser parafusada sem manipulação vigorosa através do orifício criado com o introdutor; deve-se ter atenção para que a âncora não seja posicionada sob a cartilagem articular e sim sob o osso subcondral; $4^{\circ}$ ) Os cirurgiões devem lembrar-se de que a manipulação vigorosa durante a inserção da âncora pode inadvertidamente fazer com que sua extremidade fique sem corte e isso pode tornar impossível desparafusá-la. Se a âncora estiver malposicionada ou deslocada durante a cirurgia artroscópica, ela deve ser retirada.

Nossa constatação após a avaliação desses 28 pacientes nos faz concordar com as afirmações acima. Entretanto, no que se refere à retirada das âncoras colocadas inadequadamente, quando notamos que as tentativas insistentes podem causar danos ainda maiores à cartilagem glenoide adjacente à âncora, optamos por sepultá-la.

Tem-se proposto o uso de âncoras absorvíveis como alternativa para minimizar as complicações, porém, sabe-se que a resistência inicial observada com âncoras bioabsorvíveis tem sido menor e o período de absorção não é inferior a um ano.

Foi verificado em estudos radiográficos, tomando-se como referências as variações do diâmetro dos orifícios de inserção de âncoras absorvíveis em 10 pacientes e, com o seguimento máximo de 38 meses, que os sinais de substituição por osso só foram observados a partir de um ano de pós-operatório ${ }^{(11)}$. Entretanto, com seis meses observa-se que a resistência é semelhante, tanto com âncoras absorvíveis, como não absorvíveis ${ }^{(12)}$.

Pesquisadores concordam que a resistência da sutura com âncoras está relacionada principalmente com a qualidade do osso no qual a âncora está inserida e, secundariamente, depende da resistência do fio de sutura, dos nós e da qualidade dos tecidos reparados.

Atualmente, existe maior pressão econômica sobre o uso de âncoras bioabsorvíveis, em função de seus altos custos. Deve-se salientar que o erro com as âncoras radiotransparentes (bioabsorvíveis) é menos evidente, o que pode retardar o diagnóstico do mau posicionamento da âncora, o tratamento e piorar significativamente o prognóstico.

\section{CONCLUSÃO}

O posicionamento inadequado das âncoras metálicas de suturas em artroscopia de ombro é o fator essencial nas complicações decorrentes das mesmas.

\section{REFERÊNCIAS}

1. Rhee YG, Lee DH, Chun IH, Bae SC. Glenohumeral arthropathy after arthroscopic anterior shoulder stabilization. Arthroscopy. 2004;20(4):402-6.

2. Koss S, Richmond JC, Woodward JR. Two to five-year follow up of arthroscopic bankart reconstruction using a suture anchor technique. Am J Sports Med. 1997;26(6): 809-12.

3. Kaar TK, Schenck RC Jr, Wirth MA, Rockwood CA Jr. Complications of metallic suture anchors in shoulder surgery: a report of 8 cases. Arthroscopy. 2001;17(1):31-7

4. Zuckerman JD, Matsen FA 3rd. Complications about the glenohumeral joint related to the use screws and staples. J Bone Joint Surg Am. 1984; 66(2):175-80.

5. Hawkins RJ, Bokor DJ. Clinical evalution of shoulder problems. In: The Shoulder. Philadelphia : Saunders; 1998. p.168-92.

6. Amustutz HC, Sew Hoy AL, Clarke IC. UCLA anatomic total shoulder arthroplasty. Clin Orthop Relat Res. 1981;(155):7-20.
7. Outerbridge RE. The ethiology of chondromalacia patellae. J Bone Joint Surg Br. 1961;43:752-7.

8. Samilson RL, Prieto V. Dislocation arthropathy of the shoulder. J Bone Joint Surg Am. 1983; 65(4):456-60.

9. Patte D, Debeyre J. Luxations récidivantes de l’épaule. Eneyel Med Chir Techn Chirurg Orthop . 1982:44:265.

10. Ejnisman B, Andreoli CV, Pochini AC, Monteiro GC, Feloppa F, Cohen M. Artropatia glenoumeral pós-tratamento de lesões labiais com implantes metálicos. Rev Bras Ortop. 2006;41(5):167-72

11. Warme WJ, Arciero RA, Savoie FH 3rd, Uhorchak JM, Walton M. Nonabsorbable versus absorbable suture anchors for open bankart repair a prospective, randomized comparison. Am J Sports Med. 1999;27(6):742-6.

12. Barber FA, Herber MA, Richards DP. Sutures and suture anchors: update. Arthroscopy. 2003;19(9):985-90. 